\title{
Getting it right first time-implications from a survey of current intra-ocular lens availability for cataract surgery across the West of Scotland
}

\author{
Manaim Shah ${ }^{1} \cdot$ David Lockington $\mathbb{1}^{1}$
}

Received: 28 March 2020 / Revised: 9 April 2020 / Accepted: 15 April 2020 / Published online: 28 April 2020

(c) The Royal College of Ophthalmologists 2020

Most cataract surgeries are completed successfully with planned in-the-bag intra-ocular lens (IOL) placement. However, if surgery becomes complicated due to posterior capsule rupture (PCR), additional surgical adjuncts, techniques and other IOL options may be required (but only useable if available at that point in time). PCR rates have been quoted as occurring in every 50 cases (1.95\%), so PCR is a relatively uncommon but predictable situation, and the frequency can be reduced by appropriate risk stratification and case selection [1]. As NHS units are being encouraged to increase the volume of surgical throughput by delivering routine cataract surgery within 30 minutes of theatre time per case, it follows that equipment levels to address potential complications should equally increase to match this demand [2]. In light of this, we wished to survey the IOL options, power range and stock levels available to surgeons on the day of surgery across NHS ophthalmic theatre units in the West of Scotland, and identify policies regarding consent and back up IOL options.

All ten theatre units responded to our survey in autumn 2019. All stocked one-piece IOLs with overall ranges from -5 to $+40 \mathrm{D}$ (minimum quantity 0 ; maximum 20 ; mean 3 ; median 3). The most commonly used IOLs (power +18 to $+26.5 \mathrm{D}$ ) had an overall mean quantity of 8 (median 8 ) across this sub-group. The more extreme IOL powers beyond that range had variably limited availability (quantity 0-5). Nine theatre units stocked 3-piece IOLs (range +2 to $+30 \mathrm{D}$; minimum quantity 0 ; maximum 5 (mean 2 ; median 2)). At least one lens was present for the common powers at these nine surgical units $(+12$ to $+23.5 \mathrm{D})$.

David Lockington

davidlockington@hotmail.com

1 Tennent Institute of Ophthalmology, Gartnavel General Hospital, 1053 Great Western Road, Glasgow G12 0YN, UK
Beyond this range, there was variably limited availability (quantity 0-3). Six theatre units stocked anterior chamber IOLs $(+5$ to $+30 \mathrm{D}$; whole dioptre intervals; minimum quantity 1 ; maximum 3 (mean 1 ; median 1)). Only one theatre unit stocked iris clip IOLs $(+10$ to $+25 \mathrm{D}$, whole dioptre intervals; minimum quantity 2 ; maximum 2 (mean 2; median 2)) [See Table 1].

All ten theatre units had a policy to order an additional back up IOL for special order IOLs (toric/multifocal/ extremes of range) which is consistent with NICE guidelines [3]. However, no surgical unit had a policy to order an additional appropriately powered 3-piece IOL in case of complications resulting in the requirement for sulcus placement in these potentially complex cases. No department had any stated policy to leave such patients aphakic and plan secondary IOL insertion and there was no clear documentation of this circumstance being discussed as part of the routine consent process.

Our survey reveals that NHS ophthalmic operating theatres have arbitrary IOL stock levels, which are limited in choice for extreme powers. We could not identify any publications advising how to safely determine IOL stock levels as a proportion of surgical activity, which is a concern in the current climate of 'lean surgical practice'. Equipment stock levels appear to be based on previous usage, on an ad-hoc 'use and replace' basis in conjunction with the various IOL companies who manufacture and/or distribute the IOLs. This could lead to sub-optimal management if an intra-operative complication is encountered, if a 1-piece IOL cannot be placed in the bag, and there is no appropriately powered back up IOL available. This situation may become more frequent in the drive to make surgical lists more productive through larger volumes and in locations with multiple concurrent theatre lists. Equipment shortages could also be exacerbated at a time when NHS operating theatres are increasingly and additionally being utilised at the weekend by external cataract providers. NICE 
Table 1 Table illustrating median stock levels of different intra-ocular lenses (IOLs) across ten ophthalmic units in the West of Scotland, showing good availability of commonly used single-piece IOLs ( 15.0 to $26.5 \mathrm{D}$ ) and 3-piece IOL (10.0 to $27.5 \mathrm{D})$, but limited variability at the extremes, and marked limitations of both range and quantity for Anterior Chamber IOLs (ACIOL) and iris clip IOLs. The density of shading reflects greater availability of IOL stock.

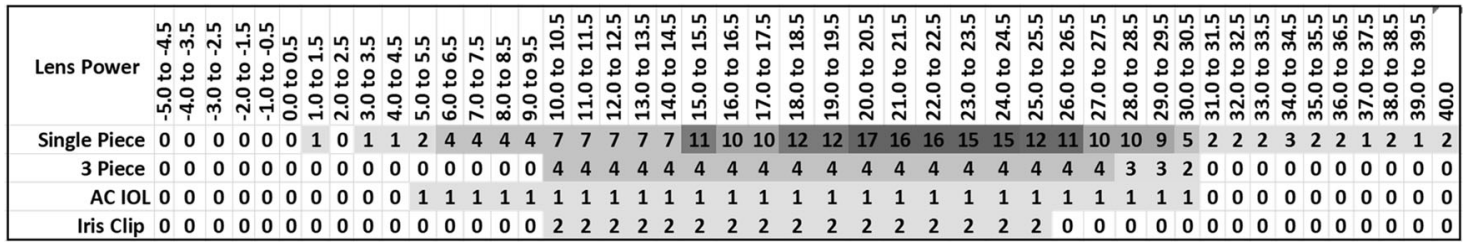

guidelines to prevent wrong IOL errors state there should be 'only 1 intra-ocular lens in the theatre, that matches the person's selected lens type and prescription, that at least 1 additional identical intra-ocular lens is in stock, and that alternative intra-ocular lenses are in stock in case the selected lens needs to be changed if there are complications during surgery' [3]. Our survey results would suggest that the potentially required alternative IOLs are not always available, particularly at the extremes of IOL power range.

This survey also confirms the clinical impression that the availability and power range of ACIOLs is diminishing, and due to associated endothelial complications and the renewed interest in scleral IOL fixation, may become obsolete [4, 5]. In light of this, we recommend that the potential for deferred secondary IOL implantation should be included in the consent process to address the aim of the NICE recommendations. In addition, the necessary equipment levels for IOLs and surgical adjuncts should be addressed and maintained appropriately to ensure all cataract patients experience the best possible outcomes through adherence to the Get It Right First Time principles [2].

Acknowledgements A version of this study was a poster presentation at the Scottish Ophthalmological Club meeting, February 2020.

\section{Compliance with ethical standards}

Conflict of interest The authors declare that they have no conflict of interest.

Publisher's note Springer Nature remains neutral with regard to jurisdictional claims in published maps and institutional affiliations.

\section{References}

1. Day AC, Donachie PH, Sparrow JM, Johnston RL, Royal College of Ophthalmologists' National Ophthalmology Database. The Royal College of Ophthalmologists' National Ophthalmology Database study of cataract surgery: report 1 , visual outcomes and complications. Eye. 2015;29:552-60.

2. MacEwen C, Davis A, Chang L. Ophthalmology GIRFT Programme National Specialty Report. 2019. https://gettingitrightfirsttime.co. uk/wp-content/uploads/2019/12/OphthalmologyReportGIRFT19PFINAL.pdf. Accessed 27 Mar 2020.

3. NICE guideline [NG77]. Cataracts in adults: management. 2017. https://www.nice.org.uk/guidance/ng77. Accessed 27 Mar 2020.

4. Jacob S, Kumar DA, Rao NK. Scleral fixation of intraocular lenses. Curr Opin Ophthalmol. 2020;31:50-60.

5. Holt DG, Young J, Stagg B, Ambati BK. Anterior chamber intraocular lens, sutured posterior chamber intraocular lens, or glued intraocular lens: where do we stand? Curr Opin Ophthalmol. 2012;23:62-7. 